\title{
TUMBLING INSTABILITY IN OBLATE ROTATING STELLAR SYSTEMS
}

\author{
E. SZUSZKIEWICZ, J.C.B. PAPALOIZOU, A. J. ALLEN and P.L. PALMER \\ School of Mathematical Sciences, Queen Mary $\mathcal{E}$ Westfield College, London
}

ABSTRACT. We present evolutionary calculations which show the tumbling instability found in spherical stellar systems by Allen et al.(1992) also occurs in some initially oblate rotating systems.

\section{Introduction}

Allen et al. (1992) have recently presented calculations for spherical systems with some degree of rotation obtained by reversing the $z$ component of angular momentum, $J_{z}$, of some fraction of the stars. These showed a non-axisymmetric instability which resulted in a slowly tumbling triaxial bar with ratio of major to minor axis being about 1.5. In view of the potential importance of this instability for explaining the structure of elliptical and the bulges of spiral galaxies, we have extended these calculations to a variety of initially axisymmetric oblate rotating models to study the range of parameter space for which the instability occurs as well as the range of possible end states.

\section{Initial models}

The distribution function of the initial models was taken to be the sum of three components in the form

$$
f=c_{1}\left(E_{01}-E\right)^{n_{1}}+c_{2}\left(E_{02}-E+\omega J_{z}\right)^{n_{2}}+c_{3}\left|J_{z}\right|^{k}\left(E_{03}-E\right)^{n_{3}+(k+3) / 2} .
$$

Here $k, E_{0 i}, c_{i}, n_{i}(i=1,2,3)$ and $\omega$ are constants. This leads to a mass density in cylindrical coordinates $(r, z)$ given by

$\rho=d_{1}\left(E_{01}-\phi\right)^{n_{1}+3 / 2}+d_{2}\left(E_{02}-\phi-\frac{1}{2} \omega^{2} r^{2}\right)^{n_{2}+3 / 2}+d_{3} r^{k}\left(E_{03}-\phi\right)^{n_{3}+(k+3) / 2}$,

where $d_{i}, i=1,2,3$ are new constants proportional to the $c_{i}$ and $\phi$ is the gravitational potential. The first contribution is that of an isotropic polytrope, and the second corresponds to a polytrope rotating with constant angular velocity $\omega$. The $E_{0 i}$ are chosen to limit the extent of any contribution to a fixed fraction of the surface equatorial radius and the $d_{i}$ are chosen to fix the relative masses in the various contributions. The models which are invariant to reversing $J_{z}$ for any number of stars are constructed by an iterative procedure and more details will be published elsewhere. As an illustrative example, we present evolutionary calculations for a model which contained $\frac{1}{3}$ of its mass in the first component with the remainder in the third with $k=1$, the first extending up to $\frac{1}{2}$ of the full equatorial radius. The $z$ component of angular momentum of $\frac{3}{4}$ of the particles was then reversed.

\section{Numerical Results}

The initial axisymmetric model had an axial ratio of 1.35 and was followed for 550 crossing times using the low noise numerical technique described in Allen et al. (1990). Here the basic time unit of one crossing time $\equiv 0.41\left(G M R^{-3}\right)^{-1 / 2}$, $M, R$, and $G$ being the total mass, equatorial radius and gravitational constant respectively. After about 150 crossing times the model developed a strongly triaxial 
structure with ratio of major to minor axis of about 1.5. Figure 1 shows the axial ratios as a function of time. The rotating bar-like pattern had a period of 13 crossing times initially but this increased to about 35 crossing times by the end of the run. The rotational velocity curve at the end of the run is shown in figure 2 . At this stage the model had ratio of maximum rotational velocity to dispersion velocity of about 0.5 and ellipticity of about 0.3 , values which are typical of those found for bulges of spirals (Kormendy, 1981). There was some increase in the central condensation of the model the final density being $\propto r^{-2}$ over about two orders of magnitude.
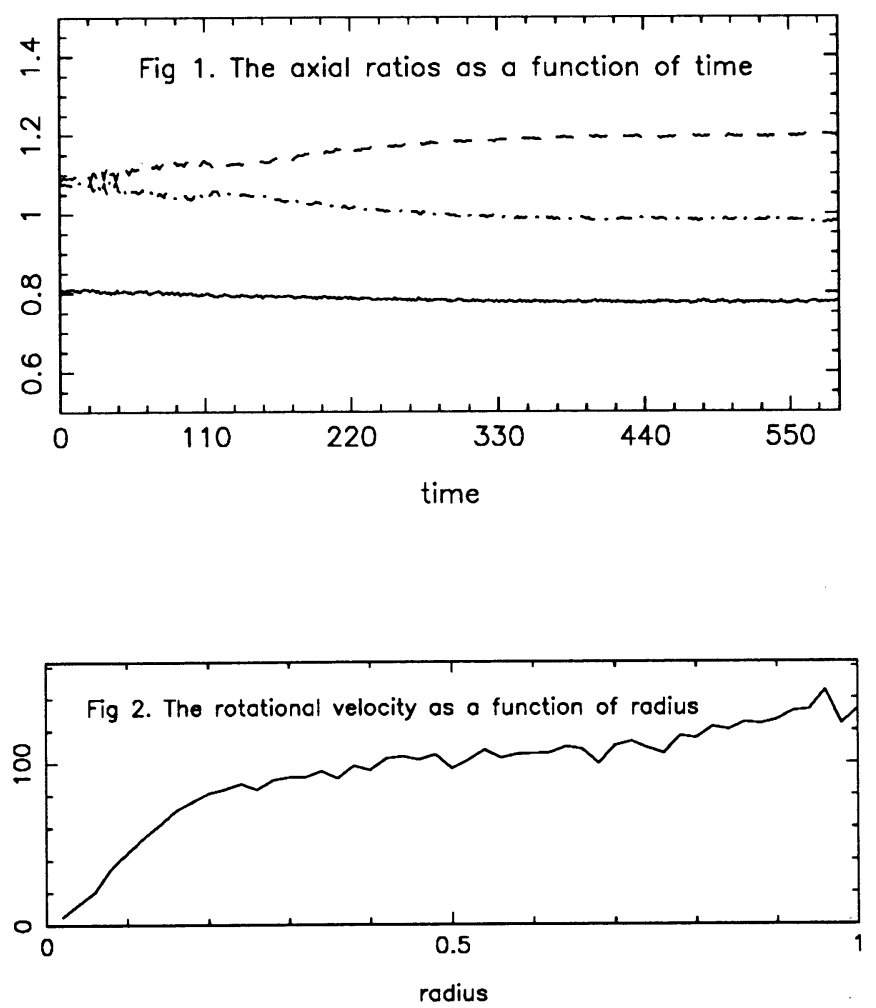

Although this model exhibited a tumbling instability similar to that found in Allen et al. (1992) not all oblate rotating models show this instability. For example uniformly rotating isotropic polytropes seem to be stable. More details of this work will be published elsewhere.

\section{References}

Allen, A.J., Palmer, P.L. \& Papaloizou, J., 1990. Mon Not R astr Soc,242, 576. Allen, A.J., Palmer, P.L. \& Papaloizou, J., 1992. Mon Not R astr Soc,256, 695. Kormendy, J., 1981. The Structure and Evolution of Normal Galaxies ed. Fall, S. M. \& Lynden-Bell, D. , Cambridge University Press.

Papaloizou, J., Palmer, P.L. \& Allen, A.J., 1991. Mon Not R astr Soc, 253, 129 . 